\title{
BioNSi: A Discrete Biological Network Simulator Tool
}

\author{
Amir Rubinstein ${ }^{1}$, Noga Bracha ${ }^{1}$, Liat Rudner ${ }^{1}$, Noga Zucker $^{1}$, Hadas E. \\ Sloin $^{2}$, and Benny Chor ${ }^{1}$ \\ ${ }^{1}$ Blavatnik School of Computer Science, Tel Aviv University \\ ${ }^{2}$ Department of Neurobiology, George S. Wise Faculty of Life Sciences and Sagol \\ School of Neuroscience, Tel Aviv University
}

Supporting Information:

In this file (in order of appearance in the main text):

- Supplementary 1: the biological rationale behind the structure and parameters of the designed computational networks

- Figure S-1 - The $\Delta$ Clock mutation.

- Figure S-2 - A hypothetical effect of light on the circadian clock.

- Supplementary 2: An example for the construction of a "toy" network based on given data

- Figure S-3 - A "toy" network and its stepwise construction according to biological data.

As separate files:

- CYSNetworks --- the networks that appear as case studies in .cys format.

- CSVNetworks --- the networks that appear as case studies in .csv format. 


\section{Supplementary 1: the biological rationale behind the structure and}

\section{parameters of the designed computational networks}

We provide a detailed justification for the structure and specific parameters of the computational networks used in the second case study - the circadian clock network. By "structure of the network", we mean the exact nodes and edges that comprise it, including dependency edges. By "network parameters", we refer to weights and delays on edges, initial states of node, and the pre-set pattern of timed nodes. All our choices were guided by biological knowledge and previous findings about these specific systems. When knowledge was partial, the corresponding parameters were chosen based on trial and error process.

1. Nodes and Edges

The network describes the molecular mechanism of the vertebrate circadian clock. It contains nodes representing known components of the main and accessory circadian clock loop [1], [2]. Components of the main loop include Clock, Bmal1, Per, and Cry, as well as the protein CK1. Genes that have multiple copies, such as Per and Cry, are represented by only one copy for simplification. Components of the accessory loop include Dec1, Rev-erba and ROR, and were selected based on their known role and possible involvement in the connection to the TGF- $\beta$ signaling system [3]. Two additional nodes represent the mRNA and protein levels of clock controlled genes (CCGs), and serve as output components of the clock. For each of these components, separate nodes represent mRNAs, proteins, heterodimers in the cytosol, and heterodimers inside the nucleus. Heterodimers were separated to these two components because of the time delay between the dimerization process and the 
entrance of the dimer to the nucleus, where it acts as a transcription factor. The weights and delays of all the edges appear in the table below. Nodes' states change through the simulation under the regime of the transition function, with two exceptions: (1) the CLOCK node, and (2) the CK1 node. The Clock node was chosen to remain at a fixed state throughout simulations, since its mRNA was shown to exhibit no circadian oscillations in the mammalian central clock [4]. CK1 was not previously shown to be regulated by any other components represented in our network. In the beginning of the simulation, the initial state of most clock components was set to 0 , except CLOCK (on a fixed level of 3), CK1 (on a fixed level of 3), and Bmal1 mRNA (which was set on a level of 9 in order to initiate the first cycle).

We added to our network components of the TGF- $\beta$ signaling system in order to depict the connection between the circadian clock molecular mechanism and TGF- $\beta$ signaling. They include TGF- $\beta$ protein (representing either TGF- $\beta 1 / 2 / 3$ ), Smad3 mRNA, Smad3 protein, phosphorylated Smad3 (pSmad3) pSmad3:Smad4 complex, Smad7 mRNA, and Smad7 protein. Components were represented by two separate nodes for mRNA and protein only when they undergo direct or indirect regulation by the circadian clock, like Smad3 (that is regulated by Clock:Bmal1) and Smad7 (that is regulated by pSmad3:Smad4 complex). We did not include a separate node for Smad4 mRNA and protein for reasons of simplicity, since its activity is not of special importance to the connection between the two systems. To represent the dependency of the phosphorylation of Smad3 on TGF- $\beta$ activity, the node between Smad3 and pSmad3 is dependent on TGF- $\beta$ (more information on dependencies is explained later on). Because our system does not include any components that 
regulate the activity of TGF- $\beta$ itself, its levels were fixed in the beginning of the simulation to 0 (absence of TGF- $\beta$ ) or 9 (presence of TGF- $\beta$ ).

2. Edges weights, delays and dependencies

Each edge connecting two nodes in the system has two attributes: a weight, which corresponds to the strength of the regulation, and a delay, which corresponds to the time it takes this interaction to occur. The default weight for a positive regulation is +1 and for negative regulation -1 . Cases in which the interaction is considered stronger were set to different values, as detailed below. The default value for delays was 0 (no delay), while interactions that are either delayed, or have relatively long duration are represented by higher delay values, as described below. The edges weight and delays are set as follows:

- Edges from an mRNA node to the corresponding protein node, representing translation, are assigned with weight $=+2$ and delay $=3$. A weight higher than the default was chosen for these edges since one mRNA molecule can be translate into multiple protein molecules. A delay $=3$ was chosen to represent the duration of the process of translation from mRNA to active protein.

- Edges between transcription factor to an mRNA are assigned weight=+2 and delay=1. A higher weight was chosen for these edges since one transcription factor can influence multiple mRNA molecules. Delay $=1$ was chosen to represent the duration of the process of transcription.

- Edges from protein to heterodimer are set to weight $=+2$ and delay $=0 . \mathrm{A}$ weight higher than the default was chosen for these edges to emphasize the 
necessity of the represented dimers activity for the circadian clock molecular mechanism.

- Each mRNA has a self-degradation edge with weight $=-1$ and delay $=0$. Each protein has a self-degradation edge with weight $=-2$ and delay $=0$. These edges represent the fact that both mRNA and proteins in the cell degrade over time. Because most mRNAs tend to be translated into proteins before they degrade, they were assigned a smaller degradation value than their protein counterparts.

Another type of interaction in the model takes the form of dependency between components. In our networks, we introduced three types of dependent interactions:

- In dimerization (see Clock:Bmal1 or Per:Cry in Figure S-1), there is a crossdependency between the two monomers: each monomer protein is dependent on the other protein's levels in order to increase the levels of ("transform into") the dimer.

- In the activity of transcription factors (Clock:Bmal1 or pSmad3:Smad4 complex), the activity of the transcription factor is dependent on the levels of negative regulators. For example, high levels of Per:Cry or Dec1 interfere with the ability of Clock:Bmal1 to upregulate transcription. Because Per:Cry is considered to be part of the main loop of the circadian clock, while Dec1 only part of the accessory loop, the negative dependency of Dec1 is weaker than of that of Per Cry (in order for Clock:Bmal1 to up-regulate mRNA levels Dec1 has to be lower than 8 but Per:Cry lower than 2).

- The phosphorylation process in which Smad3 becomes pSmad3 is dependent on TGF- $\beta$ levels. 
All Edges weight, delays and dependencies in the designed BioNSi networks

Self-regulatory edges were always of -1 weight and 0 delay for mRNA and of -2 weight and 0 delay for protein and are not included in the table.

\begin{tabular}{|c|c|c|c|c|}
\hline \multicolumn{5}{|c|}{ The molecular vertebrate circadian clock network } \\
\hline Source Node & Target node & Weight & Delay & Dependencies \\
\hline Bmal1 & BMAL1 & 2 & 3 & - \\
\hline BMAL1 & CLOCK:BMAL1 C & 2 & 0 & CLOCK $>0$ \\
\hline CLOCK & CLOCK:BMAL1 C & 2 & 0 & BMAL $>0$ \\
\hline CLOCK:BMAL1 C & CLOCK:BMAL1 N & 1 & 3 & - \\
\hline CLOCK:BMAL1 N & $\operatorname{ccg}$ & 2 & 1 & $\mathrm{DEC}<8, \mathrm{PER}: \mathrm{CRY}<2$ \\
\hline CLOCK:BMAL1 N & per & 2 & 1 & DEC $<8$, PER:CRY $<2$ \\
\hline CLOCK:BMAL1 N & cry & 2 & 1 & DEC $<8$, PER:CRY $<2$ \\
\hline CLOCK:BMAL1 N & dec1 & 2 & 1 & $\mathrm{DEC}<8, \mathrm{PER}: \mathrm{CRY}<2$ \\
\hline CLOCK:BMAL1 N & rev & 2 & 1 & $\mathrm{DEC}<8, \mathrm{PER}: \mathrm{CRY}<2$ \\
\hline CLOCK:BMAL1 N & ror & 2 & 1 & $\mathrm{DEC}<8, \mathrm{PER}: \mathrm{CRY}<2$ \\
\hline $\operatorname{ccg}$ & CCG & 2 & 3 & - \\
\hline per & PER & 2 & 3 & - \\
\hline cry & CRY & 2 & 3 & - \\
\hline Dec1 & DEC1 & 2 & 3 & - \\
\hline rev & REV & 2 & 3 & - \\
\hline ror & ROR & 2 & 3 & - \\
\hline PER & PER:CRY C & 2 & 0 & $C R Y>0$ \\
\hline CRY & PER:CRY C & 2 & 0 & PER $>0$ \\
\hline PER:CRY C & PER:CRY N & 1 & 4 & - \\
\hline PER:CRY N & Bmal1 & 2 & 2 & - \\
\hline DEC1 & Bmal1 & 2 & 1 & - \\
\hline REV & Bmal1 & -1 & 2 & - \\
\hline ROR & Bmal1 & 1 & 2 & - \\
\hline CK1 & PER & -1 & 0 & - \\
\hline \multicolumn{5}{|c|}{ The TGF- $\beta$ signalling connection to the circadian clock network } \\
\hline CLOCK:BMAL1 N & smad3 & 2 & 1 & $\mathrm{DEC}<8, \mathrm{PER}: \mathrm{CRY}<2$ \\
\hline smad3 & SMAD3 & 2 & 3 & - \\
\hline SMAD3 & pSMAD3 & 2 & 2 & TGFB $>5$, SMAD7<5 \\
\hline pSMAD3 & pSMAD3:4 & 2 & 2 & - \\
\hline pSMAD3:4 & dec1 & 1 & 1 & - \\
\hline pSMAD3:4 & Smad7 & 1 & 1 & - \\
\hline smad7 & SMAD7 & 2 & 3 & - \\
\hline
\end{tabular}




\section{References}

[1] C. H. Ko and J. S. Takahashi, "Molecular components of the mammalian circadian clock," Hum. Mol. Genet., vol. 15, no. SUPPL. 2, pp. 271-277, 2006.

[2] G. Vatine, D. Vallone, Y. Gothilf, and N. S. Foulkes, "It's time to swim! Zebrafish and the circadian clock," FEBS Lett., vol. 585, no. 10, pp. 1485-1494, 2011.

[3] N. Kon, T. Hirota, T. Kawamoto, Y. Kato, T. Tsubota, and Y. Fukada, “Activation of TGFbeta/activin signalling resets the circadian clock through rapid induction of Dec1 transcripts.," Nat. Cell Biol., vol. 10, no. 12, pp. 1463-9, Dec. 2008.

[4] P. L. Lowrey and J. S. Takahashi, “Mammalian circadian biology: elucidating genomewide levels of temporal organization.," Annu. Rev. Genomics Hum. Genet., vol. 5, no. 47, pp. 407-441, 2004. 


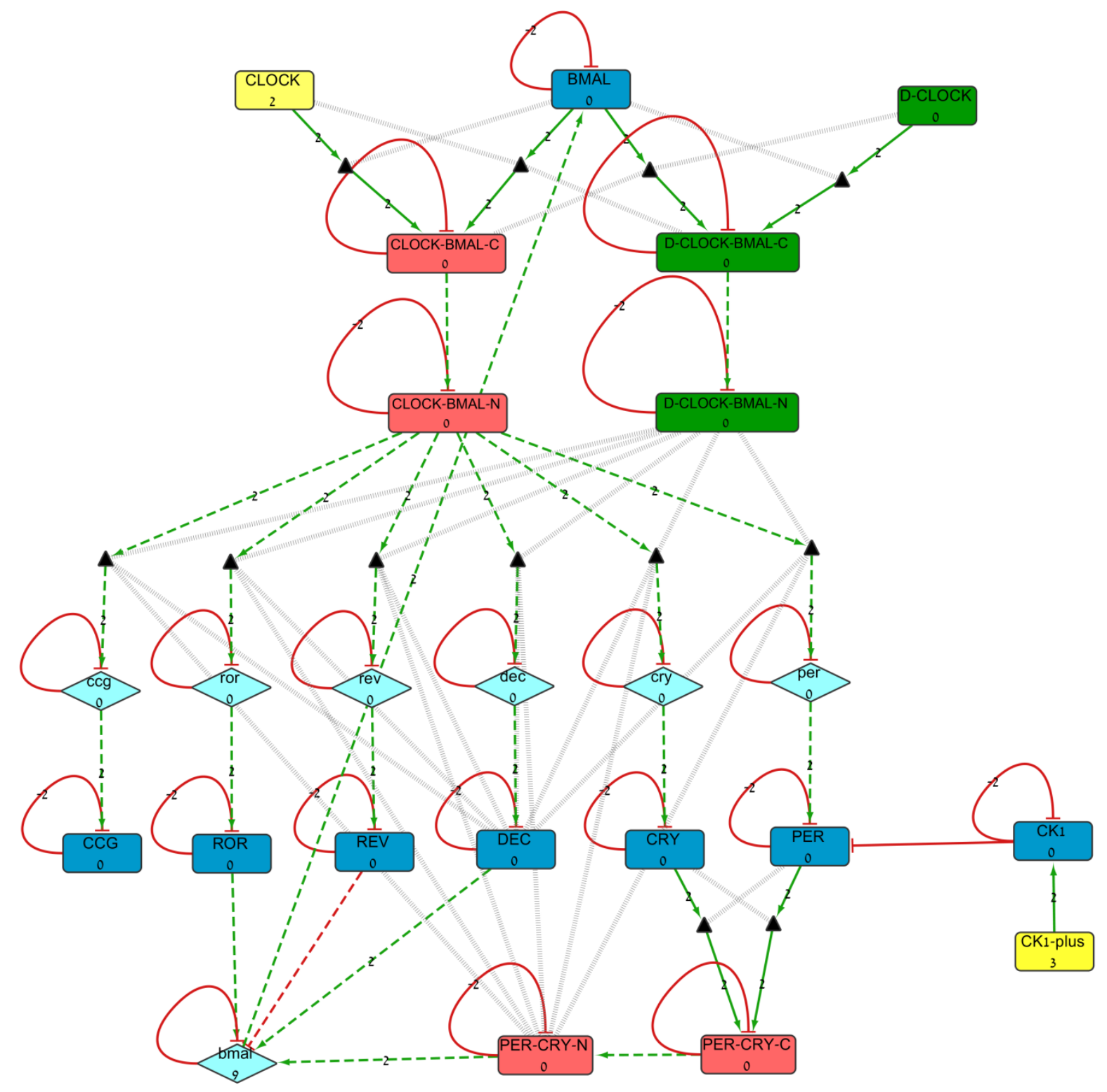

Figure S-1 - The $\Delta$ Clock mutation.

The circadian clock network with the addition of three nodes representing the effect of the $\triangle$ Clock mutant: D-CLOCK, D-CLOCK-BMAL-C and D-CLOCK-BMAL-N. 


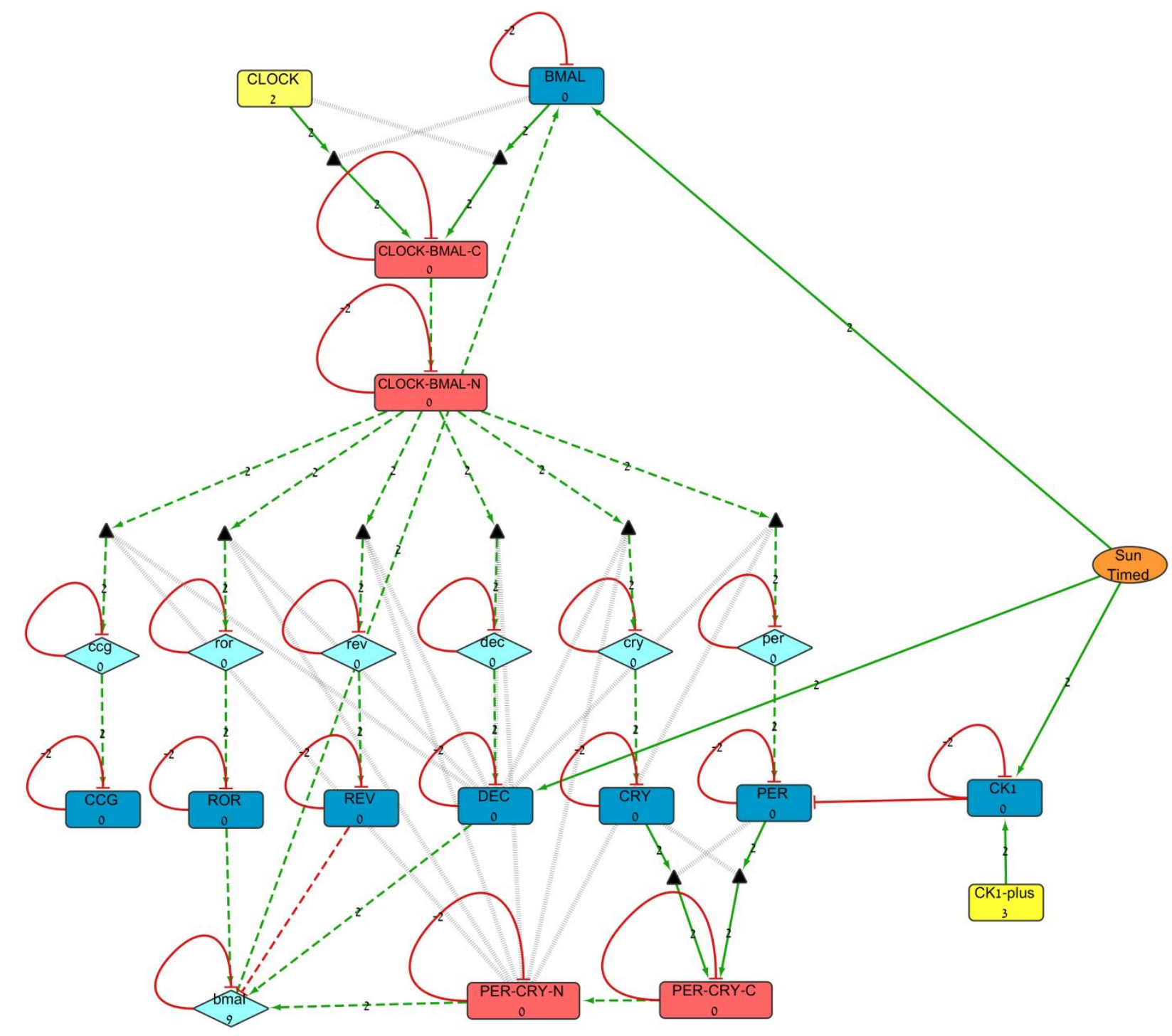

Figure S-2 - A hypothetical effect of light on the circadian clock.

The circadian clock network with the addition of a "Sun" node representing light. 


\section{Supplementary 2: An example for the construction of a "toy" network based on given data}

Suppose you know of three genes A, B and C, which regulate each other in a negative loop structure: At the beginning of the biological process, A is present at high level and activates $B, B$ activates $C$, and $C$ down-regulates $A$. Fig. S-3a shows a representation of this simple network. The initial state of $A$ is 9 , and that of $B$ and $C$ is 0 . Simulation of this network shows a decline in the level of $A$, while $B$ followed by C increase their levels and stay up. Suppose experimental data does not agree with these results. We will now exemplify two modifications to the network.

Scenario 1: suppose experimental data shows a decline in the level of A, followed by a transient expression of $B$ and then $C$. In the end of the biological process all three genes are shut down. Looking at the results in Fig. S-3a, we see that $\mathrm{A}$ indeed declines, but B and C do not shut down after they are expressed. The reason is that nothing represses their level in the network. At this point we should think what represses these genes in "reality" and update the network accordingly. If for example we merely know that the product of these genes is degraded over time, we should add negative self-loops to their nodes. Fig. S-3b shows the outcome of this modification. Alternatively, we may want to introduce into the network additional nodes, representing e.g. known proteins that down-regulate B and C. Note that dependency edges that block the activation of $\mathrm{B}$ or $\mathrm{C}$ will not be enough here, as the mere lack of activation will not cause a decline in a node's state. 
Scenario 2: Suppose, in addition, that experimental data shows a cyclical expression pattern, in which $A, B$ and $C$ go up and down sequentially. Fig. S-3b shows that all the genes are shut down at the end. The reason is that the network does not have any source for up-regulation that should reactivate the genes. Let us add an additional mode, denoted "signal" that represents the biological or chemical entity that reactivates the system. We initialize this new node with state 1 , to denote that the signal is active. Since no other node regulates this signal node, it will remain in state 1 throughout the simulation (for more advanced behavior types for signals, such as single pules, cyclic expression etc., use the "timed node" option, described later). The network and simulation outcome is shown in Fig. S-3c. 

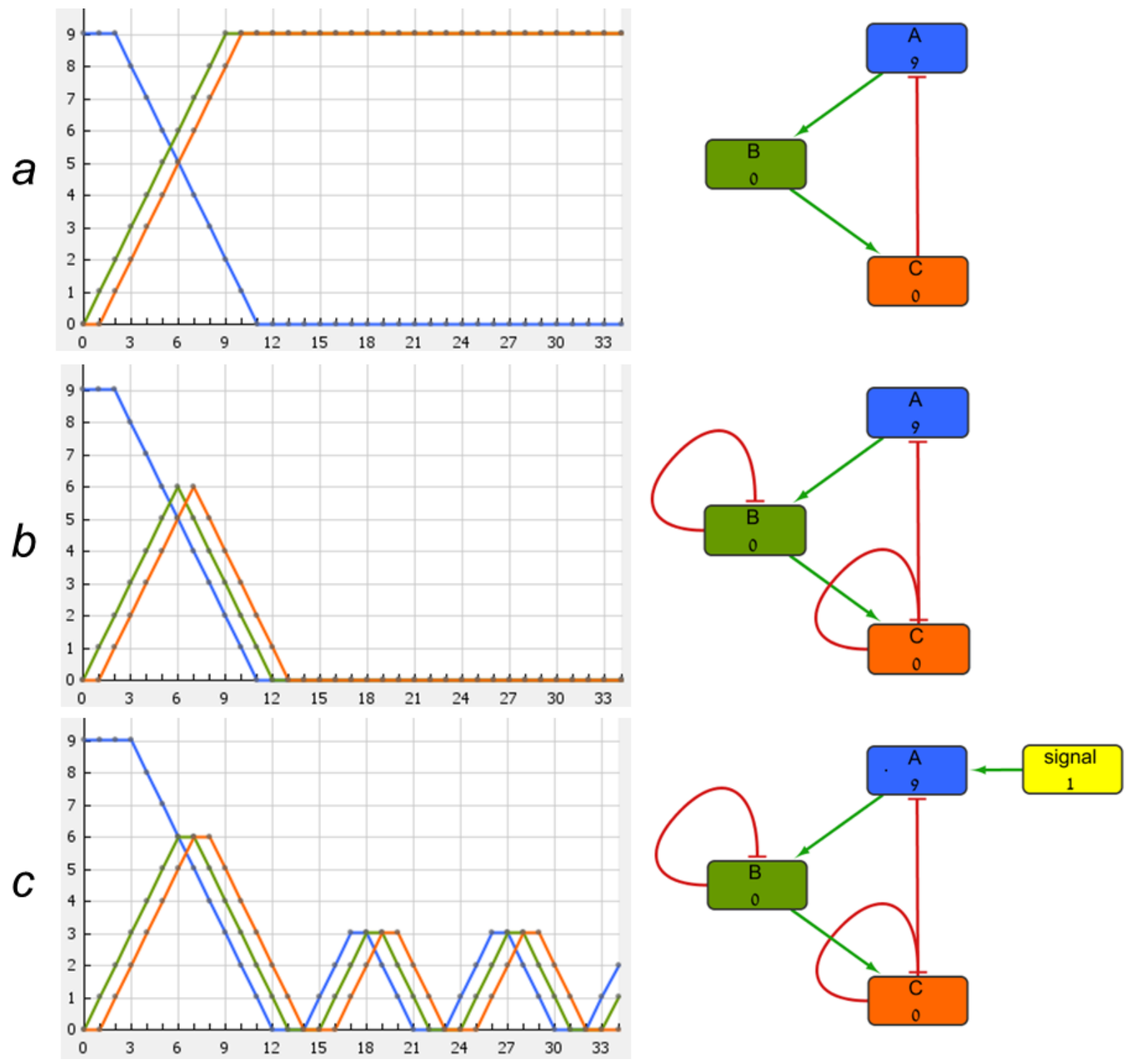

Figure S-3 - A "toy" network and its stepwise construction according to biological data. 